\title{
Educação permanente visando à integralidade do cuidado na rede de saúde mental
}

\author{
Permanent education for the integrality of care in the mental health network \\ Educación permanente para la integralidad del cuidado en la red de salud mental
}

Recebido: 03/01/2021 | Revisado: 04/01/2021 | Aceito: 09/01/2021 | Publicado: 09/01/2021

\author{
Silvia Cristina Pereira dos Santos \\ ORCID: https://orcid.org/0000-0002-1612-3334 \\ Universidade Federal Fluminense, Brasil \\ E-mail: silviacps56@gmail.com \\ Elaine Antunes Cortez \\ ORCID: https://orcid.org/0000-0003-3912-9648 \\ Universidade Federal Fluminense, Brasil \\ E-mail: nanicortez@hotmail.com \\ Geilsa Soraia Cavalcanti Valente \\ ORCID: https://orcid.org/0000-0003-4488-4912 \\ Universidade Federal Fluminense, Brasil. \\ E-mail: geilsavalente@gmail.com \\ Suellen Gomes Barbosa Assad \\ ORCID: https://orcid.org/0000-0002-4911-3837 \\ Universidade Federal Fluminense, Brasil \\ E-mail: suellengomesbarbosa@gmail.com
}

\begin{abstract}
Resumo
Objetivo: Identificar as dificuldades enfrentadas pelos trabalhadores da rede de saúde mental e realizar ações de educação permanente, fomentando a discussão sobre o movimento de estruturação da Rede de Atenção Psicossocial. Método: Pesquisa-ação com aplicação de questionário e realização de oficinas de educação permanente com 22 trabalhadores da rede de saúde mental de Japeri, RJ, cujos dados foram tratados por análise de conteúdo temática. Resultados: Foram analisadas quatro categorias: Desafiando gigantes: estrutura do trabalho na saúde mental; "Despreparo" profissional e a construção na reflexão da prática: a grande virada; Articulação: integralidade e intersetorialidade, caminho para romper as desigualdades; Desdobramentos da pesquisa e das oficinas de Educação Permanente em Saúde: a reflexão na ação gerou construção. Conclusões e implicações para a prática: A partir do processo de reflexão-na-ação, os entraves que fragmentam os serviços de saúde mental foram analisados, permitindo a elaboração de propostas coletivas de intervenção que promovam o cuidado integral aos usuários.
\end{abstract}

Palavras-chave: Saúde mental; Educação permanente; Integralidade em saúde; Pessoal de saúde; Intersetorialidade.

\begin{abstract}
Aim: To identify the difficulties faced by workers in the mental health network and to carry out permanent education activities, encouraging discussion on the movement of the Psychosocial Attention Network. Method: Action research with questionnaire application and realization of permanent education workshops with 22 workers of the mental health of Japeri, RJ, whose given were treated by analysis of thematic content. Results: were analyzed four categories: Defying giants: structure of work in mental health; Professional "unprepared" and the construction in the reflection of the practice: the large turn; Articulation: integrality and intersectoriality, a way to break inequalities; Unfolding of the research and the health permanent education workshops: reflection in action generated construction. Conclusions and implications for practice: from reflection-in-action process, barriers that fragments mental health services were analyzed, allowing the elaboration of collective proposals for intervention that promote the integral care to users.

Keywords: Mental health; Education, Continuing; Integrality in health; Health personnel; Intersectoral collaboration.

\section{Resumen}

Objetivo: Identificar las dificultades que enfrentan los trabajadores en la red de salud mental y llevar a cabo actividades de educación permanente, fomentando la discusión sobre el movimiento de la Red de Atención Psicosocial. Método: Investigación de acción con aplicación de cuestionario y realización de talleres de educación permanente con 22 trabajadores de la salud mental de Japeri, RJ, cuyos resultados se trataron mediante análisis de contenido temático. Resultados: Se analizaron cuatro categorías: Desafiando gigantes: estructura del trabajo en salud mental; Profesional "despreparado" y la construcción en el reflejo de la práctica: el gran giro; Articulación: integralidad e intersectorialidad, una forma de romper las desigualdades; Desdoblamientos de la investigación y de los talleres de educación permanente en salud: la reflexión en la acción generó construcción. Conclusiones e implicaciones para la práctica: A partir del proceso de reflexión en acción, se analizaron las barreras que fragmentan los servicios de salud mental, permitiendo la elaboración de propuestas colectivas de intervención que promuevan la atención integral a los usuarios.
\end{abstract}


Palabras clave: Salud mental; Educación continua; Integralidad en salud; Personal de salud; Colaboración intersectorial.

\section{Introdução}

Há 17 anos foi aprovada a Lei no 10.216, conhecida como a Lei da Reforma Psiquiátrica Brasileira, que instituiu um novo olhar ao usuário com transtorno mental e, por isso, caracteriza-se como marco após vários movimentos de luta de profissionais, usuários e familiares em prol de um novo modelo assistencial de saúde mental (Brasil, 2001; Pitta, 2011).

Apesar dos avanços no cenário assistencial do País verificados nos primeiros anos de instituição da referida lei, em que já era possível perceber seu potencial indutor de transformações, verificou-se a necessidade de articular, em rede, os serviços de saúde mental, com vistas à integralidade da assistência e à ampliação dos diferentes pontos de atenção para atender às pessoas com sofrimento ou transtorno mental e com necessidades decorrentes do uso de crack, álcool e outras drogas.

Nesta direção, por meio da Portaria no 3088 (Brasil, 2011) foi instituída a Rede de Atenção Psicossocial (RAPS), que estabelece suas diretrizes organizativas e de implementação em todo o País, integrando a saúde mental em todos os níveis de atenção no Sistema Único de Saúde (SUS), e orientando para o estabelecimento de ações intersetoriais que garantam a integralidade do cuidado (Santos, Pessoas Junior \& Miranda, 2018).

A propositura de construção de uma rede de serviços articulada, eficaz e humanizada desencadeou novos debates e desafios para a Política Nacional de Saúde Mental, em cujo contexto se encontram usuários com as mais diversas subjetividades que definem práticas profissionais nem sempre coerentes com as demandas da população e com as políticas de saúde (Nunes, Guimarães \& Sampaio, 2016), o que implica a necessidade de promover mecanismos de educação permanente aos trabalhadores que nela operam.

Essa problemática e a dificuldade de articulação da rede que se observa na práxis, no que se refere à assistência com os usuários de transtorno mental do município de Japeri, RJ, foi o que levou as autoras a desenvolverem o presente estudo. Parte-se do pressuposto que é a prática em serviço que busca novos caminhos para o cuidado que, influenciados pelo movimento da Reforma Psiquiátrica, visam à melhoria da qualidade de vida dessa população.

A Educação Permanente em Saúde (EPS), alçada como política pública em 2004, é entendida como aprendizagem baseada na problematização do processo de trabalho em saúde e tem por objetivo transformar as práticas e a organização do trabalho (França, Belisário \& Medeiros, 2018). Nesse sentido, assume-se a EPS como um importante instrumento de reflexão das práticas em saúde mental, com vistas à promoção do cuidado integral aos usuários da RAPS (Leite, Rocha \& Santos, 2018).

Pelo exposto, este artigo objetivou identificar as dificuldades enfrentadas pelos trabalhadores da rede de saúde mental e realizar ações de educação permanente com base nessas dificuldades, fomentando a discussão sobre o movimento de estruturação da RAPS.

\section{Metodologia}

Trata-se de estudo descritivo-exploratório, de abordagem qualitativa, do tipo pesquisa-ação. Teve como cenário a rede de saúde mental do município de Japeri, Baixada Fluminense, RJ, que vinha buscando articular a rede com o objetivo de trabalhar de forma intersetorial as ações de cuidado em saúde mental, conforme orienta as diretrizes da RAPS.

Entende-se por métodos qualitativos aqueles nos quais o pesquisador interpreta com sua opinião o fenômeno em estudo (Pereira, Shitsuka, Parreira, \& Shitsuka, 2018).

A pesquisa foi desenvolvida em duas fases: a exploratória e a de ação. Na exploratória foi utilizado um questionário autoaplicável, com questões referentes ao perfil do respondente e três questões abertas, que objetivou diagnosticar a realidade 
contextual da rede de saúde mental do município, por meio do levantamento das dificuldades enfrentadas pelos trabalhadores da RAPS no cotidiano do trabalho, e captar sugestões que apoiassem o planejamento de ações de EPS na rede.

A fase de ação consistiu na realização de nove oficinas de EPS com trabalhadores da RAPS, nas quais se deu o processo de ação-reflexão da própria prática, com vistas a repensar as dificuldades da RAPS, tendo por base três casos (situação de aprendizagem) derivados das respostas do questionário aplicado na primeira etapa.

Para determinação da amostra, estabeleceu-se como critérios de inclusão: trabalhadores da saúde do município, que atuassem em algum ponto da rede de saúde mental de modo articulado ao Centro de Atenção Psicossocial II, local onde funciona a Coordenação de Saúde Mental e ocorre as reuniões semanais de equipe multiprofissional com atores de diferentes dispositivos. Foram excluídos os profissionais que perderam o vínculo com o município, que não participaram da reunião de equipe no período em que foi aplicado o questionário e que faltaram em mais de duas oficinas de EPS (fase de ação).

A amostra foi composta, então, por 22 trabalhadores, representantes do CAPS, Residência Terapêutica, Equipe de Desinstitucionalização, Polo Álcool e outras drogas, Polo Infanto Juvenil, Atenção Básica e Coordenação do Programa de Saúde Mental.

Os dados foram coletados no horário destinado à reunião de equipe no CAPS II, em outubro de 2014. As respostas contidas nos questionários autoaplicáveis foram digitadas e organizadas de modo a constituir o corpus de análise. Os resultados dessa fase possibilitaram o planejamento das oficinas de EPS, que ocorreram em novembro e dezembro de 2014, também no CAPS II, cujas discussões foram registradas em diário de campo.

Para a análise dos dados do questionário, este estudo encontrou sustentação nos preceitos metodológicos da análise de conteúdo, que pressupõe três etapas. Na etapa de pré-análise se procedeu com a leitura do material a ser tratado. Na de exploração ou codificação, as mensagens dos sujeitos foram decompostas em 157 unidades de registros (UR) referentes às dificuldades estruturais, sociais e pessoais enfrentadas pelos trabalhadores da RAPS de Japeri. Agrupadas, essas UR originaram 17 unidades de significado (US) que, por sua vez, foram reunidas nos seguintes eixos analíticos: estrutura da rede; qualificação profissional; e integralidade da atenção.

Essa análise inicial desencadeou a realização das oficinas de EPS, nas quais as dificuldades evidenciadas na fase exploratória serviram de base para a elaboração de três casos de aprendizagem, trabalhados durante as oficinas a partir do método de problematização, com a finalidade de refletir sobre as situações-problemas e propor intervenções.

Os resultados das oficinas foram reunidos aos da primeira fase (exploratória), agrupando-os nos três eixos analíticos iniciais, que compuseram as seguintes categorias temáticas: "Desafiando Gigantes": estrutura do trabalho na saúde mental; "Despreparo" profissional e a construção na reflexão da prática: a grande virada; e Articulação: integralidade e intersetorialidade, o caminho para os rompimentos. Ademais, uma categoria foi acrescida para a descrição dos produtos do processo desencadeado por esta pesquisa, qual seja: Desdobramentos da pesquisa e das oficinas de EPS: a reflexão na ação gerou construção.

Por fim, na última etapa da análise, os dados foram interpretados à luz do referencial teórico de Donald Schön (2009) e Paulo Freire (2007).

O estudo foi desenvolvido cumprindo os preceitos éticos que visam assegurar os direitos dos sujeitos participantes de pesquisas, seguindo a Resolução n466/2012, que regulamenta a pesquisa que envolve seres humanos. Desse modo, cumprindo o ritual protocolar, o projeto da pesquisa foi apreciado e aprovado pelo Comitê de Ética da Faculdade de Medicina da Universidade Federal Fluminense - UFF, CAAE: 33725114.3.0000.5243, Parecer Consubstanciado $\mathrm{n}^{\circ}$ 843.827. Os participantes assinaram o Termo de Consentimento Livre e Esclarecido, bem como foram informados sobre os riscos existentes na pesquisa. 


\section{Resultados}

A pesquisa incluiu 22 trabalhadores que atuam com usuários de saúde mental na RAPS de Japeri, RJ, dos quais 17 (77,3\%) mulheres. A idade variou entre 20 e 34 anos, com média de 40,5 anos. O grupo foi conformado por nove profissionais com formação superior na área da saúde (enfermeiros, psicólogos e farmacêuticos); seis que atuam como técnicos ou em funções auxiliares de saúde (auxiliares e técnicos de enfermagem, cuidadores, oficineiros); e sete com funções administrativas ou de apoio (motoristas, recepcionista, serviços gerais, segurança e assistentes administrativos).

\section{$1^{a}$ Categoria: "Desafiando gigantes": estrutura do trabalho na saúde mental}

Esta categoria foi construída com base em $98(62,4 \%)$ UR, destacadas dos questionários aplicados na fase exploratória, que se centram nas dificuldades com relação à estrutura física e organizacional, escassez de insumos básicos, má gestão, dificuldades de acesso e número reduzido de pessoal, que impossibilitam o acolhimento digno dos usuários e a condução das ações de saúde.

Falta de material para atendimento mínimo para farmácia e oficinas (E3).

Espaço inadequado para atendimento de crianças e adolescentes (E5).

Falta de água, luz; tem infiltração e vazamentos no CAPS (E11).

Não há apoio nem investimento do nosso prefeito (E13).

Falta de funcionário, falta de médico (E23).

Nas oficinas de EPS, a partir da discussão dos casos de aprendizagem, essas dificuldades foram ratificadas, e a hipótese levantada para esse problema foi: a falta de investimentos dos gestores no fortalecimento de recursos humanos, na compra de insumos e na infraestrutura a fim de melhor adequar a rotina dos serviços.

Nesse movimento, os profissionais chegaram à conclusão de que precisavam organizar o Conselho Gestor do CAPS, uma vez que esse, junto ao conselho municipal, poderia fazer valer o controle social, já que em diversos aspectos a má gestão aparece como um entrave para a estruturação e organização do serviço. Assim sendo, as questões estruturais nesse cenário da saúde mental foram consideradas os "gigantes" a serem enfrentados pelos profissionais que, de forma corajosa, adentraram por esse 'terreno pantanoso' em busca de soluções estratégicas. Tal atitude possibilitou o empoderamento que talvez nem eles soubessem que tinham guardado dentro de si mesmos.

Diante das dificuldades encontradas, verifica-se a importância da formação do Conselho Gestor através de eleição, potencializando a saúde mental frente às solicitações na Secretaria de Saúde. Faz-se necessário também a aproximação dos gestores de saúde e do Secretário de Saúde nas reuniões de colegiados e fóruns. E a gestão precisa ser entendida como ação política, com interlocução, avaliação, pluralidade e interdisciplinaridade, sendo esta descentralizada, com a participação da comunidade, visando à cidadania ampliada (Reflexão coletiva elaborada pela equipe durante as oficinas).

\section{$2^{a}$ Categoria: "Despreparo profissional" e a construção na reflexão da prática: a grande virada}

Das respostas dos questionários foram destacadas $21(13,4 \%)$ UR relativas ao sentimento de despreparo profissional para o enfrentamento de algumas situações clínicas, não conhecimento da RAPS, frustração e insegurança.

Conhecimento específico para manejar alguns diagnósticos graves (E5).

Profissionais não treinados/qualificados na área da saúde mental (E11).

Falta de conhecimento da RAPS e suas diretrizes (E18). 
Ao participarem das oficinas e se aproximarem da proposta de EPS, os trabalhadores identificaram que há necessidade de qualificação profissional, conforme se verifica na reflexão coletiva elaborada pela equipe durante as oficinas, após discussão dos casos de aprendizagem:

A qualificação contínua das equipes é uma demanda que se apresenta de maneira emergencial, tanto em relação a uma formação mais aprofundada sobre determinados assuntos quanto para uma formação ampliada a partir dos princípios da EPS, que levem a uma ação técnica mais assertiva, estabelecendo limites pessoais e profissionais na atuação profissional (Reflexão coletiva elaborada pela equipe durante as oficinas).

\section{$3^{a}$ Categoria: Articulação: integralidade e intersetorialidade, o caminho para os rompimentos}

Verificou-se que há desarticulação entre os serviços e ausência de comunicação entre os serviços da rede, que dificultam a integralidade e a intersetorialidade. O preconceito e o estigma também foram evidenciados como atitudes que obstam o acesso do usuário aos serviços do SUS. Esse retrato ficou constatado a partir de 38 (24,2\%) UR recortadas dos questionários que apontam para a problemática do cuidado integral.

O preconceito e despreparo da rede com a saúde mental (E3).

A rede de atenção é falha, desestruturada, não integrada (E11).

Identifico uma imensa dificuldade no estabelecimento da intersetorialidade (E15).

Isolamento dos diversos setores (E16, E21).

A falta de compreensão da sociedade quanto ao usuário em crise, dos familiares que não os ajudam a melhor desenvolverem-se (E22).

Durante as oficinas, as hipóteses levantadas para essas questões se relacionam à ausência de espaços de discussões intersetoriais para formulação de fluxos e estratégias de intervenção, estreitando, assim, as relações entre os serviços para melhorar a qualidade da assistência e a efetiva integralidade da atenção.

O acesso à rede pelo usuário de saúde mental passa pela ótica do empoderamento do usuário, com o fortalecimento de sua capacidade de ser responsável também por seu tratamento. [...] esse circuito engloba a informação, seja através do investimento, articulação e difusão, e necessita de uma constante retroalimentação das experiências no que se refere a uma melhor integralidade dessa rede composta de diversos atores, que pode ser realizada através de fóruns intermunicipais, acolhimento e orientação às famílias, promoção de ações territoriais envolvendo a comunidade, estruturação de equipes de matriciamento, colegiados e política de educação permanente promovendo a articulação de rede, levando a desmitificação e preconceitos referentes à loucura (Reflexão coletiva elaborada pela equipe durante as oficinas).

\section{Desdobramentos da pesquisa e das oficinas de Educação Permanente em Saúde: a reflexão na ação gerou construção}

\section{a) Colegiado de Gestores da Rede de Atenção Psicossocial e cartilha informativa}

Após a primeira etapa da pesquisa (fase exploratória), constatou-se a necessidade de sensibilizar os gestores dos serviços da RAPS sobre a importância da integração em saúde e da Política de Educação Permanente em Saúde, fomentando a discussão sobre o movimento de estruturação da saúde mental no município e a liberação dos trabalhadores para a participação no processo de ESP (oficinas).

Assim, um dos pesquisadores, juntamente com a Coordenação de Saúde Mental do município, organizou quatro encontros, em outubro e novembro de 2014, com os gestores dos serviços da RAPS, que recebeu a denominação de Colegiado de Gestores da RAPS.

No primeiro encontro do colegiado, que recebeu o título "De um emaranhado de opiniões, tecemos uma rede de contribuições", os participantes chegaram à conclusão de que pouco se sabia sobre o Programa de Saúde Mental do município 
e propuseram a organização de uma cartilha sobre a estruturação e o fluxo da rede de saúde mental, para ser divulgada junto à população nos serviços da rede. Nos três encontros posteriores, deu-se a construção coletiva da cartilha intitulada pelos gestores como: Saúde Mental: "Desconstruir para Construir". Cumpre destacar que foi acordada a continuidade do colegiado, a partir de encontros mensais.

\section{b) Conselho Gestor do Centro de Atenção Psicossocial}

No movimento de discussão, os profissionais chegaram à conclusão de que precisavam organizar o Conselho Gestor do CAPS, já que este sedia os polos e a equipe de desinstitucionalização, sendo também responsável por acompanhar os moradores das RT. Nestes termos, ficou decidido que nas próximas reuniões de equipe seria discutida e organizada a composição para eleição do conselho.

\section{c) Mesa redonda}

Constatada a necessidade de expandir a discussão sobre os problemas enfrentados na RAPS de Japeri, foi organizada uma mesa redonda com profissionais convidados e representante dos usuários, sugeridos pela equipe, realizada durante a oitava oficina de EPS. Durante esse encontro, cujo tema definido foi "Educação Permanente para promover a integralidade da rede de saúde mental", todos os debates evidenciaram a necessidade da rede em desenvolver o trabalho integral, a importância da gestão pública participativa e a importância da RAPS.

\section{d) Portfólio e documentário sobre o processo de Educação Permanente em Saúde na Rede de Atenção Psicossocial de Japeri}

A fim de elucidar o processo de implementação da Política de Educação Permanente da RAPS de Japeri, foi construído um portfólio embasando teoricamente o desenvolvimento das oficinas e traçando o caminho ascendente feito pelos profissionais envolvidos. E, com o objetivo de documentar, ampliar e difundir a proposta sobre o referido processo, um documentário foi desenvolvido, lançado na rede mundial de computadores.

\section{e) Continuidade da Educação Permanente em Saúde}

Ao final da última oficina, os trabalhadores da rede elaboraram uma proposta de continuidade da EPS, constando os profissionais responsáveis pelos próximos encontros, o tempo de duração, os locais, os objetivos, a periodicidade e o públicoalvo.

\section{Discussão}

Neste estudo, os profissionais vivenciaram a partir da reflexão na ação o caos estrutural, que retoma a ideia de Schön (2009) sobre terreno pantanoso como espaço no qual os problemas confusos desafiam as soluções. Nele, o profissional de saúde precisou adentrar para analisar e ponderar, através do pensamento reflexivo, quais as estratégias possíveis de resolução do caos em que se encontra. O movimento de reflexão-na-ação proposto pelo referido autor foi feito pelos profissionais deste estudo durante as oficinas, cujo sentimento de empoderamento foi sendo construído em cada encontro, possibilitando construir propostas resolutivas para os problemas do "pântano"(Neto, Silva \& Rua, 2018).

Autores constataram que a composição de espaços de discussão, que demarcam um processo de articulação com diferentes pontos de atenção da RAPS no território, promovem a interlocução entre os serviços, configurando a 
corresponsabilidade na execução da atenção em saúde (Leite, Rocha \& Santos, 2018; Haberland, Pavam, Cruz, Joia, Oliveira \& Rocha, 2017), e o aprendizado e construção coletiva de modos de enfrentamento das dificuldades que se apresentam.

Neste sentido, Freire (2011) defende a educação como uma forma de intervir na realidade e, com ela, aprender e empreender mudanças reais na sociedade. E o que movimenta toda e qualquer transformação nos serviços de saúde é, na verdade, a inquietação com o instituído, que demanda novas estratégias de formular ações, integrando o cuidado na assistência (Leite \& Rocha, 2017).

E pensando em cuidado, apresenta-se uma reflexão a respeito do cuidado em saúde mental e a fineza do cuidado esvaziado de saber prévio, que se vale do não-saber, que não se institui por meio de padronizações generalizantes e generalizáveis por se voltar ao singular do sujeito, uma vez que estamos lidando com vidas. Trata-se de um cuidado “artesanal”, algo feito um a um (Kurimoto, Moreira \& Bertuol, 2017).

Para isso são criadas novas políticas que fortalecem a proposta do cuidado integral, com viés nessa construção coletiva de EPS, como estabelece, por exemplo, as diretrizes para o funcionamento da RAPS, definindo os cuidados de forma articulada entre os serviços e reafirmando a importância da formação permanente para promover a qualidade na assistência prestada (Kinker, Moreira \& Bertuol, 2018).

Neste contexto, autores pontam a importância do cuidado integrado entre os serviços, estando estes organizados e realmente funcionando em rede, com propostas de ações em consonância com as Unidades Básicas de Saúde, Estratégia da Saúde da Família, o Centro de Atenção Psicossocial, e demais dispositivos que compõem a RAPS (Pereira et al., 2020).

Por outro lado, para obter a aplicação dos recursos na assistência de forma adequada, faz-se necessário o controle social de maneira efetiva, visando à gestão compartilhada do SUS e a viabilidade em atender as demandas dos serviços de saúde. E serão os segmentos envolvidos que deverão participar da tomada de decisão nas políticas que são definidas no seu nível de atuação, fortalecendo o preceito de democratização da política de saúde (Fernandes, Moreira \& Ribeiro, 2016).

Schön (2009), em sua teoria de prática reflexiva, incentiva um profissional mais ativo, autônomo, consciente em relação as suas opções. O processo de reflexão-na-ação pode ser desenvolvido numa série de momentos. No primeiro, o da surpresa, o profissional reflexivo permite-se ser surpreendido; no segundo momento, ele pensa sobre o que e sobre como foi surpreendido e, em seguida, busca compreender a razão pela qual foi surpreendido; em outro momento, denominado pelo autor como terceiro momento, reformula o problema acendido pela situação; e, no quarto momento, executa uma experiência a fim de testar a sua nova hipótese. Neste contexto, o profissional poderá desenvolver o processo de reflexão-na-ação, para o qual é possível olhar retrospectivamente e refletir.

Do mesmo modo, Freire (2011) apresenta a educação como um caráter permanente, pois para ele o saber se faz de forma constante, por isso não há seres educados e não educados, estamos todos nos educandos.

Neste sentido, verifica-se uma aproximação entre as teorias de Schön (2009) e Freire (2011), em seus escritos, dado que elas estabelecem a reflexão como eixo norteador de todo o trabalho, considerando que a formação parte da totalidade do sujeito e que nenhum conhecimento fica à margem.

Schön (2009) propõe a ideia da reflexão sobre a reflexão-na-ação da prática, que passa pelo viés do ensino prático por meio do estudo da vida organizacional dos profissionais. O autor preconiza que o conhecimento profissional poderia ser melhorado a fim de estar mais próximo de atender aos anseios sociais; para tanto, sugere que os profissionais encontrem um terreno comum onde as diferentes formas de saber possam ser usadas para resolver os problemas indeterminados da prática (Mickleborough, 2015).

Assim sendo, o ensino prático, a educação num caráter permanente e a vida organizacional estão atravessados pela mesma linha horizontal, que num movimento espiral se unem. 
Nessa perspectiva, para Schön (2009), as pessoas que exercem uma profissão são diferentes entre si, em suas subespecialidades, nas experiências e nas perspectivas particulares que trazem para seu trabalho e em seus estilos de operação, no entanto, elas compartilham de um corpo de conhecimento profissional. Desse modo, o processo de conhecer na ação tem suas raízes no contexto social e institucionalizado, em cujo âmbito uma comunidade compartilha.

Entendendo-se a importância da articulação entre os serviços que resulta na ação integral, Mattos (Mattos, 2004) aponta que de todos os princípios e diretrizes do SUS, o da integralidade talvez seja o menos visível na trajetória do sistema e de suas práticas.

Assim, trabalhar de forma articulada e integrada significa criar dispositivos e adotar processos coletivos de trabalho que permitam oferecer, para além das ações demandadas pela própria população a partir de experiências individuais de sofrimento, ações voltadas para a prevenção, promoção, tratamento e reabilitação, isto é, para o cuidado em todas as esferas.

O presente estudo evidenciou a importância da aprendizagem significativa, como afirma Freire (2011) quando diz que o conhecimento pode diminuir os danos quando se faz necessário lidar com os problemas, pois a partir daí é possível intervir na realidade e, dessa forma, ninguém pode estar no mundo, com o mundo e com os outros de forma neutra. Assim, a troca e a convivência com o outro são o que o autor chama de "escolha", "decisão" e "intervenção na realidade".

A pesquisa em voga aponta que, garantindo-se a integralidade e a intersetorialidade das políticas sociais e reduzindo a fragmentação entre os serviços, será possível reduzir as problemáticas sociais, promovendo melhor assistência à saúde.

A proposta das oficinas foi fundamentada à luz da obra de Paulo Freire, uma vez que este considera que o ato de ensinar não é transferir conhecimento, mas criar as possibilidades para a sua própria construção, a qual está diretamente ligada ao território em que as pessoas vivem (2009). Verifica-se o quanto, em meio as lutas, dificuldades e entraves que os profissionais dessa rede em questão têm vivenciado, o processo reflexivo trouxe enriquecimento e crescimento para a equipe.

Nessa mesma linha, evidencia-se que o processo vivenciado nesse estudo em questão também se fundamenta na prática reflexiva de Schön, já que ele propõe que se o profissional quiser tratar a prática de forma competente, deve fazê-lo testando estratégias situacionais que ele próprio produz (2009).

Desse modo, ao verificar os entraves que fragmentavam os serviços, buscou-se repensar a ação mediante a prática reflexiva, tomando como base a propositura de Freire (2011) de refletir sobre a "luta", no sentido de que o ato de lutar é a reinvenção da forma histórica de lutar e deve ser permanente. Pelo exposto, esta pesquisa trouxe uma compreensão do empoderamento dos trabalhadores no cenário da prática, evidenciando os aspectos que apontam para o "poder" da reflexão-naação estabelecido no espaço coletivo. Processo que resultou na possibilidade de diferenciar as dificuldades da gestão pública que apontam para as questões da estrutura organizacional, as dificuldades da qualificação profissional e a articulação entre a integralidade e a intersetorialidade, levando os sujeitos a questionarem a força do "gigante" em relação à prática e indicando resolubilidades para os problemas, distribuindo para cada um a responsabilidade das demandas encontradas, ao mesmo tempo que unificou as possibilidades de enfrentamento em uma força coletiva.

\section{Considerações Finais}

Ao "viajar" pelo processo percorrido durante esta pesquisa, percebe-se que muitos frutos advindos da reflexão coletiva, por meio da proposta da EPS, foram colhidos pelos atores participantes, dentre os quais a sistematização e reflexão dos desafios que se apresentam no cotidiano do fazer saúde mental. Dentre tantas dificuldades identificadas, os problemas estruturais foram os que se destacaram no levantamento, indicando o caos estrutural em que se encontra a RAPS do município investigado. Também foram evidenciados os nós críticos relativos à qualificação e aos sentimentos de frustração e insegurança dos profissionais, apontando para a urgência de uma aprendizagem permanente em face aos entraves da saúde mental; e, ainda, 
aos relativos à fragilidade das ações de saúde intersetoriais e integrais, o que evidencia a importância de maior articulação entre os serviços e profissionais da rede.

A partir das oficinas de EPS, os profissionais puderam refletir sobre todos os aspectos que influenciam o processo de trabalho, e buscar, através da metodologia ativa, mobilizarem-se e criar estratégias para resolubilidade dos problemas identificados, com vistas ao cuidado integral.

Pelo exposto, conclui-se que este estudo proporcionou espaços de aprendizagem, de troca e de vivência entre gestores e trabalhadores da rede, sensibilizando-os para o cuidado integral ao usuário de saúde mental, a partir do processo de reflexãona-ação instigado durante as oficinas de EPS, nas quais os trabalhadores foram protagonistas do processo de construção do conhecimento.

Como limitação do estudo aponta-se às condições orçamentárias do município e o fraco vínculo empregatício dos profissionais, muitos dos quais demitidos após as discussões iniciadas durante este estudo, tais fatores não favorecem a continuidade dos processos de educação permanente enquanto política pública, que apenas foram iniciados com este estudo. De tal modo, a condução do projeto não assegurou, necessariamente, a sustentabilidade da proposta.

As contribuições reveladas no presente artigo apontam para a importância da institucionalização da EPS nos serviços de saúde como política pública, de modo a garantir processos reflexivos nos espaços de trabalho em saúde e a articulação dos profissionais e serviços que compõem as redes de atenção do SUS, tendo como horizonte a integralidade do cuidado. Ademais, verificou-se que a EPS tem força para empoderar os coletivos, suscitar a reflexão e, por conseguinte, a intervenção na realidade do trabalho.

Espera-se que as evidências deste estudo possam alcançar outros lugares, em um processo reflexivo e imerso em relação ao cuidado integral ao usuário da RAPS e a importância da EPS enquanto política pública. Nesse sentido, recomenda-se o desenvolvimento de novos estudos que se debruçam sobre a implantação das redes de atenção à saúde nos diferentes territórios.

\section{Referências}

Brasil. Ministério da Saúde. Secretaria Executiva. Secretaria de atenção à Saúde (2004). Legislação em Saúde Mental: 1990-2004. http://bvsms.saude.gov.br/bvs/publicacoes/legislacao_saude_mental_1990_2004_5ed.pdf

Brasil. Portaria/GM nº 3.088, de 23 de Dezembro (2011). https:// www.saude.mg.gov.br

Fernandes, F. M. B., Moreira, M. R., \& Ribeiro, J. M. (2016). Survey on health ombudsman offices in the Brazilian Unified Health System. Saúde Debate [Internet]. 40(spe):201-12. http://dx.doi.org/10.1590/0103-11042016s17

França, T., Belisário, S. A., Medeiros, K. R., Pinto, I. C. M., \& Garcia, A. C. P. (2017). Limites e possibilidades das Comissões Permanentes de Integração Ensino-Serviço: percepções dos gestores. Saúde Debate [Internet]. 41(spe3):144-54. Recuperado de: http://dx.doi.org/10.1590/0103-11042017s311

Freire, P. (2011). Pedagogia da Autonomia: Saberes Necessários à Prática Educativa. São Paulo: Paz e Terra.

Haberland, R, Pavam, C, Cruz, L. S, Joia, J. H, Oliveira, A, \&Rocha, P. R (2017). The collective practices in health policies in FÓ/Brasilândia (SP): foruns,

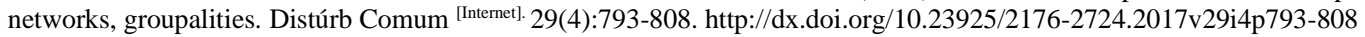

Kinker, F. S, Moreira, M. I. B, \&Bertuol, C. (2018). O desafio da formação permanente no fortalecimento das Redes de Atenção Psicossocial. Interface (Botucatu) [Internet]. Recuperado de: http://dx.doi.org/10.1590/1807-57622017.0493

Kurimoto, T. C. S., Penna, C. M. M., \&Nitkin, D. I. R. K. (2017). Knowledge and practice in mental health nursing care. Rev Bras Enferm. 70(5):973-80. http://dx.doi.org/10.1590/0034-7167-2016-0343

Leite, L. S., \& Rocha, K. B. (2017). Educação Permanente em Saúde: como e em que espaços se realiza na perspectiva dos profissionais de saúde de Porto Alegre. Estudos Psicologia. 22(2):203-13. https://dx.doi.org/10.5935/1678-4669.20170021

Leite, L. D., Rocha, K. B., \&Santos, L. M. (2018). A tessitura dos encontros da Rede de Atenção Psicossocial. Trabalho, Educação e Saúde. 16(1):183-200. http://dx.doi.org/10.1590/1981-7746-sol00101

Mattos, R. A. (2004). A integralidade na prática (ou sobre a prática da integralidade). Caderno Saúde Pública. 20(5):1411-16. http://dx.doi.org/10.1590/S0102-311X2004000500037 
Research, Society and Development, v. 10, n. 1, e23810111648, 2021

(CC BY 4.0) | ISSN 2525-3409 | DOI: http://dx.doi.org/10.33448/rsd-v10i1.11648

Mickleborough, T. (2015). Intuition in medical practice: a reflection on Donald Shön's reflective practitioner. 37(10): 889-91. http://dx.doi.org/10.3109/0142159X.2015.1078893

Netto, L., Silva, K. L., \& Rua, M. S. (2018). Reflective practice and vocational training: theoretical approaches in the field of Health and Nursing. Escola Anna Nery. 22 (1), e20170309. http://dx.doi.org/10.1590/2177-9465-ean-2017-0309

Nunes, J. M. S, Guimarães, J. M. X, \& Sampaio, J. J. C. (2016). A produção do cuidado em saúde mental: avanços e desafios à implantação do modelo de atenção psicossocial territorial. Physis. 26(4),1213-32. http://dx.doi.org/10.1590/s0103-73312016000400008

Pereira, S. S., Costa, D. R. G. O., Camargo, C. A. C. M., Rosa, W. A. G., Graciano, C. S. M., \& Ribeiro, M. I. L. C. (2020). Pet saúde interprofissionalidade: dificuldades apontadas pelas equipes de atenção básica em relação à saúde mental. Research, Society and Development, 9(7), e948975240, (CC BY 4.0) | http://dx.doi.org/10.33448/rsd-v9i7.5240

Pereira, A. S., Shitsuka, D. M, Parreira, F. J., \& Shitsuka, R. (2018). Metodologia da pesquisa científica. Ed UAB/NTE/UFSM. https://repositorio.ufsm.br/bitstream/handle/1/15824/Lic_Computacao_Metodologia Pesquisa-Cientifica.pdf?sequence=1

Pitta, A. M. F. (2011). Um balanço da reforma psiquiátrica brasileira: instituições, atores e políticas. Ciências Saúde Coletiva. 16(12):4579-89. http://dx.doi.org/10.1590/S1413-81232011001300002

Santos, R. C. A, Pessoas Junior, J. M, \& Miranda, F. A. N. (2018). Psychosocial care network: adequacy of roles and functions performed by professionals. Revista Gaúcha Enfermagem http://dx.doi.org/10.1590/1983-1447.2018.57448

Schön, D. A. (2009). Educando o profissional reflexivo: um novo design para o ensino e a aprendizagem. Penso. 